EESTI NSV TEADUSTE AKADEEMIA TOIMETISED. XII KÖIDE

FOOSIKA-, MATEMAATIKA. JA TEHNIKATEADUSTE SEERIA. 1963, NR. I

ИЗЗВЕСТИЯ АКАДЕМИИ НАУК ЭСТОНСКОЙ ССР. ТОМ ХІІ

- СЕРИЯ ФИЗИКО-МАТЕМАТИЧЕСКИХ И ТЕХННЧЕСКИХ НАУК. 1963. Ni I

\title{
К ПОЛНОМУ ОПРЕДЕЛЕНИЮ НАПРЯЖЕНИИ В СЛОИСТЫХ ФОТОУПРУГИХ мОДЕЛЯХ
}

\author{
Х. АБЕН, \\ кандидат технических наук
}

В статье доказывается, что метод, предложенный в работе [2], не позволяет определить напряжения в слонстых фотоупругих моделях. Приводятся некоторые предложения для полного определения объемного напряженного состояния при помоци слонстых моделей.

1. При экспериментальном исследовании об́ъемных задач теории упругости иногда целесообразно использовать слоистые фотоупругие модели [ $\left.{ }^{1-7}\right]$. Разработка оптически нечувствительного органического стекла $\left[{ }^{4}\right]$ значительно повысила эффективность указанного метода.

Методика исследования напряжений в фотоупругом слое совершенно аналогична исследованию замороженных срезов [8]. Однако разделение главных напряжений в слоистой модели, в общем, несколько отличается от разделения главных напряжений при методе замораживания, так как в первом случае оптические исследования позволяют определить пять компонентов тензора напряжения только в одной плоскости модели. Поэтому является актуальным исследование возможностей разделения главных напряжений при использовании слоистых фотоупругих моделей.

В статье $\left[{ }^{2}\right]$ предложен метод для разделения нормальных напряжеиий в слоистых фотоупругих моделях. Однако при разработке метода допущена ошибка, вследствие чего он является ошибочным. В настоящей статье доказывается ошибочность метода, предложенного в статье $\left[{ }^{2}\right]$, и приводятся некоторые возможности полного определения напряжений в слоистых фотоупругих моделях.

2. Как известно, методом наклонного просвечивания $\left[{ }^{9,10}\right]$ можно определить пять независимых компонентов тензора напряжения в фотоупругом слое или в замороженном срезе. В работе $\left.{ }^{2}\right]$ предлагается просвечивать фотоупругий слой в направлениях $x^{\prime}, z$ и $z^{\prime}$ (см. рис. 1; ось $y$ перпендикулярна к рисунку; оси $x$ и $y$ находятся в срединной плоскости фотоупругого слоя). Діля определения нормального напряжения $\sigma_{y}$ применяется численное интегрирование уравнения равновесия в направлении оси $y$ по формуле

$$
\sigma_{y}=\sigma_{y 0}-\sum \frac{\Delta \tau_{x^{\prime} y}}{\Delta x^{\prime}} \Delta y-\sum \frac{\Delta \tau_{y z^{\prime}}}{\Delta \varepsilon^{\prime}} \Delta y .
$$

Визникает вопрос: можно ли действительно сквозным просвечиванием фотоупругого слоя определить величины $\frac{\Delta \tau_{x^{\prime} y}}{\Delta x^{\prime}}$ и $\frac{\Delta \tau y z^{\prime}}{\Delta z^{\prime}}$ ? Рассмотрим этот вопрос подробнее. 
В общем случае объемного напряженного состояния напряжения не являются постоянными по толщине фотоупругого слоя. В первом приближении можно градиент напряжений считать линейным. Пусть некоторый компонент напряжения $\sigma_{i}$ представляется вблизи точки $O$ линейным выражением

$$
\dot{\sigma}_{i}=\sigma_{i 0}+a_{1} x+a_{2} y+a_{3} z
$$

тде $\sigma_{i 0}-$ значение $\sigma_{i}$ в точке $O, a_{i}$ - постоянные.

В этом случае изменение $\sigma_{i}$ является линейным для любого направления, например для направления просвечивания $x^{\prime}$

$$
\sigma_{i}=\sigma_{i 0}+a_{4} x^{\prime}
$$

Сквозным просвечиванием фотоупругого слоя определяются усредненные по толщине слоя величины напряжений. Интегрируя выражение (3), имеем

$$
\int_{-\frac{t^{2}}{2}}^{\frac{t^{\prime \prime}}{2}} \sigma_{i} d x^{\prime}=\sigma_{i 0} \int_{-\frac{t^{\prime}}{2}}^{\frac{t^{\prime}}{2}} d x^{\prime}+a_{4} \int_{-\frac{t^{\prime}}{2}}^{\frac{t^{\prime}}{2}} x^{\prime} d x^{\prime}
$$

Из (4) получим среднее значение напряжения. $\sigma_{i}$, определяемое сквозным просвечиванием,

$$
\frac{1}{t^{\prime}} \int_{-\frac{t^{\prime}}{2}}^{\frac{t^{\prime}}{2}} \sigma_{i} d x^{\prime}=\sigma_{i 0}
$$

Таким образом, при линейном градиенте напряжений определенные при сквозном просвечивании фотоупругого слоя напряжения соответствуют напряжениям в срединной плоскости слоя. Если же градиент напряжений является нелинейным, то при определении напряжений возникает некоторая ошибка, которая, однако, имеет значение только B местах большой концентрации напряжений [11].

Из изложенного следует, что определенные как при нормальном, так и при наклонном просвечивании фотоупругого слоя напряжения относятся к его срединной плоскости. В точках вне срединной плоскости невозможно определить напряжения сквозным просвечиванием. То же относится к исследованию замороженных срезов.

В работе [2], по-видимому, предполагается, что члены в правой части выражения (1) определяются. ссотношениями (см. рис. 1)

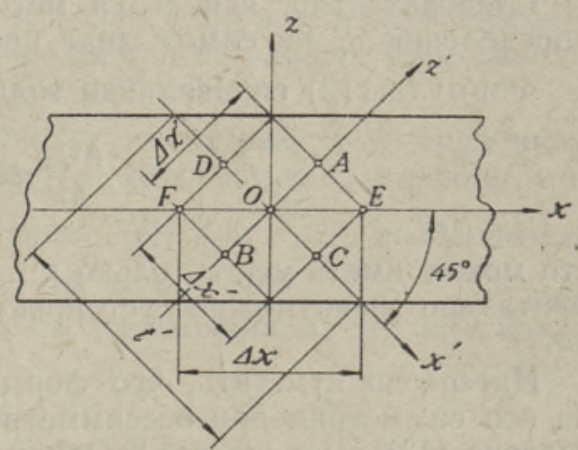

Рис. 1.

$$
\frac{\Delta \tau_{x^{\prime} y}}{\Delta x^{\prime}}=\frac{\tau_{x^{\prime} y}^{(C)}-\tau_{x^{\prime} y}^{(D)}}{\Delta x^{\prime}}, \frac{\Delta \tau_{y z^{\prime}}}{\Delta z^{\prime}}=\frac{\tau_{y z^{\prime}}^{(A)}-\tau_{y z^{\prime}}^{(B)}}{\Delta z^{\prime}} .
$$


Қак было показано выше, в точках $A, B, C$ и $D$ невозможно определить напряжения. Вместо касательных напряжений, входящих в правые части выражений (6), в действительности определяются касательные напряжения в соответствующих точках $E$ и $F$ срединной плоскости фотоупругого слоя, т. е. вместо $\tau_{x^{\prime} y}^{(C)}$ определяется $\tau_{x^{\prime} y}^{(E)}$ и т. д.

Таким образом, формулу (1) нельзя использовать для определения нормальных напряжений в слоистой фотоупругой модели, так как сквозное просвечивание не позволяет определить двух последних членов в правой части формулы (1).

Вместо величин (6) при эксперименте по методике, изложенной в статье $\left[{ }^{2}\right]$, в действительности определяются величины

$$
\frac{\tau_{x^{\prime} y}^{(E)}-\tau_{x^{\prime} y}^{(F)}}{\Delta x^{\prime}}, \frac{\tau_{y z^{\prime}}^{(E)}-\tau_{y z^{\prime}}^{(F)}}{\Delta z^{\prime}} .
$$

На основе соотношений

$$
\tau_{x^{\prime} y}=\frac{1}{2} \sqrt{2}\left(\tau_{x y}-\tau_{y z}\right), \quad \tau_{y z^{\prime}}=\frac{1}{2} \sqrt{2}\left(\tau_{x y}+\tau_{y z}\right)
$$

имеем

$$
\begin{aligned}
& \frac{\tau_{x^{\prime} y}^{(E)}-\tau_{x^{\prime} y}^{(F)}}{\Delta x^{\prime}}=\frac{1}{2} \sqrt{2} \frac{\tau_{x y}^{(E)}-\tau_{x y}^{(F)}-\tau_{y z}^{(E)}+\tau_{y z}^{(F)}}{\Delta x^{\prime}}, \\
& \frac{\tau_{y z^{\prime}}^{(E)}-\tau_{y z^{\prime}}^{(F)}}{\Delta z^{\prime}}=\frac{1}{2} \sqrt{2} \frac{\tau_{x y}^{(E)}-\tau_{x y}^{(F)}+\tau_{y z}^{(E)}-\tau_{y z}^{(F)}}{\Delta z^{\prime}} .
\end{aligned}
$$

В рассматриваемом случае имеем соотношение

$$
\Delta x^{\prime}=\Delta z^{\prime}=\frac{\Delta x}{\sqrt{2}} .
$$

На основе приведенных соображений и выражений (9)-(11) уравнение (1) приобретает вид

$$
\sigma_{y}=\sigma_{y 0}-2 \sum \frac{\Delta \tau_{x y}}{\Delta x} \Delta y
$$

Следовательно, используя методику, изложенную в статье $\left[{ }^{2}\right]$, для определения $\sigma_{y}$ на самом деле применяется формула (12).

Формула (12) справедлива только тогда, когда выполнено условие

$$
\frac{\Delta \tau_{y z}}{\Delta z}=\frac{\Delta \tau_{x y}}{\Delta x}
$$

что может иметь место только в частных случаях. Кроме того, экспериментально невозможно установить, выполнено ли условие (13) или нет.

Интересно отметить, что формула (12) оказывается справедливой на оси симметрии при осесимметричной задаче, так как в этом случае условие (13) выполняется ${ }^{1}$. Однако в этом случае для применения формулы (12) нет необходимости использовать наклонное просвечивание, так как величины (13) определяются нормальным просвечиванием.

, Ось $y$ является осью симметрнн. 
3. Рассмотрим теперь, какие возможности имеются для полного определения напряжений в слоистых фотоупругих моделях в зависимости от характера задачи.

Осесимметричная 3 ад ач а. В случае осесимметричной задачи главные напряжения можно определить численным интегрированием обобщенных уравнений Ляме-Максвелла, выведенных Джессопом $\left[{ }^{12}\right]$

$$
\frac{\partial \sigma_{1}}{\partial s_{1}}+\frac{\sigma_{1}-\sigma_{2}}{r_{2}}+\frac{\sigma_{3}-\sigma_{1}}{r_{3}} \cos \alpha=0 .
$$

Обозначения приведены на рис. 2, где $s_{i}$ - траектории главных напряжений. Можно также применять ннтегрирование уравнений равновесия в цилиндрических координатах $\left[{ }^{15}\right]$.

На оси симметрин можно использовать формулу (12).

Для определения суммы главных напряжений можно использовать численное решение уравнения Лапласа $[13,14]$

$$
\left(\frac{\partial^{2}}{\partial \mathrm{Q}^{2}}+\frac{1}{\mathrm{Q}} \frac{\partial}{\partial \mathrm{Q}}+\frac{\partial^{2}}{\partial y^{2}}\right)\left(\sigma_{1}+\sigma_{2}+\sigma_{3}\right)=0
$$

Ось о направлена перпендикулярно к оси симметрин $y$.

Плоская деформ а ия. При плоской деформации для разделения главных напряжений можно применять как численное интегрирование уравнений равновесия, так и чнсленное решение уравнения Лапласа, так как в этом случае

$$
\tau_{y z}=\tau_{z x}=0 \quad \text { пा } \frac{\partial 2}{\partial z^{2}}\left(\sigma_{1}+\sigma_{2}+\sigma_{3}\right)=0 .
$$

Плоскость сим мет р.и и. Обычно плоскость симметрии представляет для исследователя наибольший интерес. Поэтому может показаться целесообразным поставить фотоупругий слой в плоскость симметрии модели. Однако с точки зрения разделения главных напряжений это не целесообразно.

Пусть ось z перпендикулярна плоскости симметрии. Если срединная плоскость фотоупругого слоя совпадает с плоскостью симметрии модели, то невозможно определить величины $\frac{\Delta \tau_{z x}}{\Delta z}$ или $\frac{\Delta \tau_{y z}}{\Delta z}$, необходимые для численного интегрирования уравнений равновесия.

Если же фотоупругий слой вклеен на расстоянии $\Delta z$ от плоскости симметрии, то имеем

$$
\frac{\Delta \tau_{z x}}{\Delta z}=\frac{\tau_{z x}}{\Delta z}, \quad \frac{\Delta \tau_{y z}}{\Delta z}=\frac{\tau_{y z}}{\Delta z}
$$

где $\tau_{z x}, \tau_{y z}$ - касательные напряжения в фотоупругом слое (в плоскости симметрии $\tau_{z x}=\tau_{y z}=0$ ). Следовательно, целесообразно вклеить. фотоупругий слой на некотором малом расстоянии $\Delta z$ от плоскости сим- 
метрии, так как в этом случае можно наклонным просвечиванием определить все величины, необходимые для полного определения напряженного состояния.

То, что напряжения определяются не на плоскости симметрии, а на некотором расстоянии от нее, не имеет существенного значения, так как в плоскости симметрии градиент напряжений $\sigma_{x}, \sigma_{y}, \sigma_{z}$ и $\tau_{x y}$ в направлении оси $z$ равен нулю.

Общи й случа й. В общем случае объемного напряженного состояния разделить главные напряжения гораздо сложнее.

Необходимые данные для численного интегрирования уравнений равновесия можно получить, используя две одинаковые модели, в одной из которых фотоупругий слой вклеен на некотором расстоянии $\Delta z$ относительно другого.

Этот метод аналогичен разделению главных напряжений при методе замораживания, когда необходимые данные для численного интегрирования уравнений равновесия определяются нормальным и наклонным просвечиванием двух параллельных срезов $[15,10]$.

Во втором методе, предложенном Каммерером и Ламаром [16], используются также две одинаковые модели. В них фотоупругие слои поставлены на одно и то же место, но имеют различные упругие свойства.

Для разделения нормальных напряжений в слоистых фотоупругих моделях можно использовать также интерферометрический метод $\left[{ }^{17}\right]$. В этом случае достаточно иметь одну модель, однако данный метод требует специальной аппаратуры.

Из изложенного выше следует, что в случаях, когда применение слоистых фотоупругих моделей является эффективным (осесимметрич. ная задача, плоская деформация, плоскость симметрии), напряженное состояние можно определить полностью с помощью одной модели. В общем случае объемного напряженного состояния разделить нормальные напряжения сложнее, однако в этом случае в общем нецелесообразно использовать слоистые модели.

\section{ЛИТЕРА Т Р A}

1. J. E. Gibson, Stress distribution under foundations. Engineering, 180, 4680, 1955

2. Г. И. Фельдм ан, Решение объемной задачи фотоупругости при помощи слоистой модели. Вестн. Моск. ун-та, сер. мат., мех., астрон., физ., хим., 11, 1, 1956.

3. В. М. Пропк ко, Применение составных моделей для исследования объемных задач методом фотоупругости. Тр. Моск. ин-та инж. ж.-д. трансп., вып. 91, 1957.

4. Н. И. Пригоровски й, Е. Ф. Филимонова, Г. С. Дедовец, Модели для исследования напряжений пз оптически нечувствительного прозрачного материала с вклейками нз материала ЭД6-М. Заводская лаборатория, 24, 11, 1958.

5. A. K a m merer, La photo-élasticimétrie à trois dimensions. La pratique des industries mécaniques, 41, 9, 1958.

6. A. K a mmerer, A. L a ma re, La photoélasticimétrie à trois dimensions. Comptes Rendus, 246, 17, 1958.

7. В. М. Прошко, Решение объемных задач оптическим методом с применением составных моделей. Поляризационно-оптический метод исследования напряжений (тр. конференции 1958 года), Изд-во ЛГУ, 1960.

8. Х. К. А бе н, Об оптических явлениях в слонстых фотоупругих моделях. Изв. АН СССР, ОТН, механнка и машиностроение, № $5,1961$.

9. В. М. К расн ов, К решению пространственной задачи теории упругости оптипеским методом. Уч. зап. ЛГУ, серия мат. наук, № 87, 1944.

10. Х. А бе н, О применении метода наклонного просвечивания в фотоупругости. Изв. $\mathrm{AH}$ ЭССР, сер. техн. и физ.-мат. наук, 9, 1, 1960.

11. V. M. Hickson, Errors in stress determination at the free boundaries of "frozen stress" photoelastic models. British J. Appl. Phys., 3, 6, 1952. 
12. H. T. Jes s o p, The determination of the separate stresses in three-dimensional stress investigations by the frozen stress method. J. of Scient. Instrum. and of Physics in Industry, 26, 1, 1949.

13. А. К. П ре й с с, Использование метода конечных разностей для определения напряженнй на прозрачных моделях при осесимметрнчной задаче. Поляризационно-оптический метод исследования напряжений (сб. статей). Изд-во АН CCCP. 1956.

14. H. S. W o r d, J. C. W il ho it, Photoelastic analysis of the stresses around the bottom of a cylindrical bore hole due to overburden and fluid column pressure. Proc. Soc. Exp. Stress Analysis, 17, 2, 1959.

15. В. М. К а аснов, О косом просвечивании в фотоупругости. Поляризационнооптический метод исследования напряженнй (тр. конференцин 1958 года), Издво ЛГУ, 1960.

16. A. Kammerer, A. L a mare, Sur une méthode expérimentale complète pour la photoélasticimétrie à la trois dimensions. Comptes Rendus, 247, 17, 1958.

17. X. Абен, О полном определении объемного напряженного состояния методом фотоупругости. Изв. АН ЭССР, сер. физ.-мат. и техн. наук, 9, 2, 1960.

Ннститут кибернетики

Академии наук Эстонской ССР
Поступнла в редакцию

18. XII 1961

\title{
PINGEOLUKORRA TÄIELIKUST MÄÄRAMISEST KIHILISTES FOTOELASTSETES MUDELITES
}

\author{
H. Aben, \\ tehnikateaduste kandidaat
}

Resümee

Artiklis näidatakse, et töös [2] esitatud meetod ei võimalda leida pingeolukorda kihilistes fotoelastsetes mudelites ning viib vääradele tulemustele. Antakse ülevaade pingeolukorra täielikku määramist võimaldavatest meetoditest. Näidatakse, et kui optiliselt tundlik kiht asetada mitte sümmeetriatasapinda, vaid teatud väikesele kaugusele sellest, siis on kaldvalgustusmeetodil võimalik leida kōiki suurusi, mis on vajalikud pingeolukorra täielikuks määramiseks tasakaaluvōrrandite numbrilise integreerimise abil. Pingete täielikuks määramiseks ruumilise mudeli meelevaldses tasapinnas on vaja kasutada kahte mudelit, milles optiliselt tundlikud kihid on teineteise suhtes nihutatud.

\author{
Eesti NSV Teaduste Akadeemia \\ Küberneetika Instituut
}

Saabus toimetusse

18. XII 1961

\section{ON A COMPLETE DETERMINATION OF STRESS IN LAYERED PHOTOELASTIC MODELS}

\section{H. Aben}

\section{Summary}

It is shown that the method proposed in paper $\left.{ }^{2}\right]$ cannot be used for a complete determination of stress in layered photoelastic models. A review of methods enabling to separate the principal stresses in layered photoelastic models is presented. It is shown that if a photoelastic layer is not placed in the plane of symmetry, but at a little distance $\Delta z$ from it, all the terms needed for a separation of normal stresses by the shear-difference method can be determined by the method of oblique incidence. For a complete analysis of stress in any other plane, two models are needed, in which the photoelastic layers are shifted at a little distance $\Delta z$. 